Referencia para citar este artículo: Beltrán-Véliz, J., \& Osses-Bustingorry, S. (2018). Transposición didáctica de saberes culturales mapuche en escuelas situadas en contextos interculturales. Revista Latinoamericana de Ciencias Sociales, Niñez y Juventud, 16(2), 669-684. doi:https://doi.org/10.11600/1692715x.16202

\title{
Transposición didáctica de saberes culturales mapuche en escuelas situadas en contextos interculturales*
}

\author{
JUAN BELTRÁN-VÉLIZ** \\ Profesor Universidad Católica de Temuco, Chile. \\ SONIA OSSES-BUSTINGORRY*** \\ Profesora Pontificia Universidad Católica de Valparaíso, Chile.
}

\section{Artículo recibido en agosto 1 de 2017; artículo aprobado en diciembre 16 de 2017 (Eds.)}

- Resumen (descriptivo): la presente revisión del tema es de carácter descriptivo, con la cual buscamos discutir sobre la transposición didáctica de los saberes culturales mapuche que realizan los profesores y profesoras en contextos interculturales. Para llevar a cabo la revisión teórica, utilizamos las fuentes y bases de datos WOS, Thomson Reuters, SciELO, Scopus, Eric, Redalyc, Dianlet, Doaj y OEI, además de libros especializados en el tema. La revisión evidencia que los sujetos docentes requieren conocimientos pedagógicos para romper con la hegemonía de la enseñanza cultural occidental. Por lo tanto, es necesaria la utilización de procesos de transposición didáctica contextualizados, para movilizar la enseñanza de saberes científicos occidentales y saberes culturales mapuche. Esto, con el fin de favorecer la mejora continua de los procesos de enseñanzaaprendizaje y la convivencia social de los individuos estudiantes mapuches y no mapuches en contextos interculturales.

Palabras clave: educación intercultural, proceso de enseñanza, conocimientos indígenas (Tesauro de Ciencias Sociales de la Unesco).

\section{Didactic transposition of Mapuche cultural knowledge in schools located in intercultural contexts}

- Abstract (descriptive): The present review of the topic is of a descriptive nature, which seeks to discuss the didactic transposition of Mapuche cultural knowledge carried out by teachers in intercultural contexts. To carry out the theoretical review, the sources and databases used were WOS, Thomson Reuters, SciELO, Scopus, Eric, Redalyc, Dianlet, Doaj and OEI, in addition to specialized

Este es un artículo de revisión del tema. Gran área de conocimiento: Ciencias de la educación; área: Educación general; subárea: Transposición didáctica e interculturalidad (para docentes). Este estudio forma parte del marco teórico de la tesis doctoral del autor, denominada: Transposición didáctica que realizan los profesores en escuelas ubicadas en contextos interculturales en la Araucanía. Universidad de la Frontera de Temuco. Fecha de inicio de la tesis: 12 marzo de 2015, actualmente en curso. Financiada por el Programa de Doctorado de la Universidad de la Frontera, Temuco, Chile. La investigación es cualitativa.

** Profesor de Educación Básica. Licenciado en Educación. Magíster en Ciencias de la Educación, mención currículum y Evaluación. Doctor en Ciencias de la Educación por la Universidad de la Frontera, Temuco, Chile. Profesor del área de Postgrado de Educación de la Universidad Mayor de Temuco. Profesor de la Facultad de Educación, Universidad Católica de Temuco, Chile. Orcid: 0000-0003-4614-0650. Índice H5: 1. Correo electrónico: beltranovic@hotmail.com

*** Profesora de Estado en Biología y Química y Orientadora Educacional por la Pontificia Universidad Católica de Valparaíso, Chile. Magíster en Educación por la Universidad Laval, Québec-Canadá y Doctora en Educación por la Universidad Academia de Humanismo Cristiano, Chile. Directora del Magíster en Educación y Doctorado en Ciencias de la Educación, Universidad de La Frontera. Directora del Centro de Investigación y Desarrollo Educativo, Cenide. Orcid: 0000-0001-9061-7909. Índice H5: 8. Correo Electrónico: sonia.osses@ufrontera.cl 
books on the subject. The review shows that teachers require pedagogical knowledge to break with the hegemony of Western cultural education. Therefore, it is necessary to use contextualized didactic transposition processes to mobilize the teaching of Western scientific knowledge and Mapuche cultural knowledge. This, in order to favor the continuous improvement of the teaching-learning processes and the social coexistence of Mapuche and non-Mapuche students in intercultural contexts.

Key words: Intercultural education, teaching process, indigenous knowledge (Unesco Social Science Thesaurus).

\section{Transposição didáctica do conhecimento cultural mapuche em escolas localizadas em contextos interculturais}

- Resumo (descritivo): a presente revisão do tema é de natureza descritiva, que busca discutir a transposição didática do conhecimento cultural mapuche realizado por professores em contextos interculturales. Para realizar a revisão teórica, as fontes e bases de dados foram utilizadas WOS, Thomson Reuters, SciELO, Scopus, Eric, Redalyc, Dianlet, Doaj e OEI, além de livros especializados sobre o assunto. A revisão mostra que os professores precisam de conhecimento pedagógico para romper com a hegemonia da educação cultural ocidental. Portanto, o uso de processos contextualizados de transposição didática é necessário para mobilizar o ensino do conhecimento científico ocidental e do conhecimento cultural mapuche. Isto, a fim de favorecer a melhoria contínua dos processos de ensino-aprendizagem e a coexistência social dos estudantes mapuche e não mapuches em contextos interculturais.

Palavras-chave: educação intercultural, processo de ensino, conhecimento indígena (Thesaurus das Ciências Sociais da Unesco).

-1. Introducción. -2. Metodología. -3. Didáctica. -Transposición didáctica del contenido y conocimiento didáctico del contenido. -Transposición didáctica desde el contexto mapuche. -4. Consideraciones finales. - Lista de referencias.

\section{Introducción}

Uno de los grandes problemas por los que atraviesa el conflicto educativo en la actualidad se encuentra en la equidad y calidad de la educación expresada en la falta de procesos, estructura y resultados que se traduzcan en buenas prácticas y, por ende, favorezcan el aprendizaje de calidad del estudiantado. En este contexto, el rol del profesor o profesora cobra relevancia; al respecto, la Organización para la Cooperación y el Desarrollo Económico (OECD, 2005) indica que la calidad de los profesores y profesoras, y la de su enseñanza, son los factores más importantes para explicar los resultados del alumnado. En concordancia, «existe un consenso emergente acerca de que el profesorado influye de manera significativa en el aprendizaje de los alumnos y en la eficacia de la escuela» (Cochran-Smith \& Fries, 2005, p. 40). El rol del sujeto docente en el aula es determinante en la calidad del proceso de enseñanza y aprendizaje. En la misma línea, Darling-Hammond (2000) afirma que el aprendizaje de los alumnos y alumnas depende principalmente de lo que los profesores y profesoras conocen y de lo que pueden hacer.

En este contexto, desde el campo intercultural e indígena, la metodología de enseñanza utilizada en el aula queda a expensas del estilo, capacidades y creatividad que pueda tener cada profesional. Sin una formación pedagógica contextualizada y de calidad, este proceso no siempre es una instancia para el aprendizaje significativo de los estudiantes, ni para reflexionar sobre el quehacer docente. Desde esta perspectiva, y en el plano internacional y chileno, la creación de los sistemas educativos modernos durante el siglo XIX ha tenido como uno de sus ejes la consolidación de una educación monocultural, basada en una cultura dominante de corte occidental eurocéntrico (Harris, 2002; Pineau, 2001). Esto fue concebido con el objetivo de materializar la homogenización cultural y lingüística 
de las poblaciones en los territorios coloniales y al interior de los Estados nacionales (Quintriqueo, Morales, Quilaqueo, \& Arias, 2016).

Esto, en la actualidad no ha cambiado, dado que la educación que se entrega en contextos interculturales en la actualidad, conserva prácticas educativas monoculturales, provocando que se perpetúen las incomprensiones sobre la forma de abordar la diversidad social, cultural y sobre todo lingüística (Akkarai, 2009; Gordon-Burns \& Cambell, 2014; Horst \& Gitz-Johansen, 2010). Estas incomprensiones se encuentran definidas por un sistema de enseñanza y aprendizaje que se caracteriza por los siguientes aspectos: (i) el aprendizaje se encuentra distanciado del contexto sociocultural del estudiantado, lo que significa que la enseñanza y aprendizaje tiene lugar en la sala de clases y no fuera de ella; (ii) el rol de los sujetos docente y estudiante se encuentra definido de manera rígida y asimétrica, donde el profesional tiene una figura de autoridad, y es poseedor del conocimiento y de la verdad absoluta e inmutable, y el individuo estudiante es considerado un ignorante que carece de conocimientos previos; $\mathrm{y}$ (iii) los métodos educativos que utilizan se fundamentan en el conocimiento occidental, donde se ignoran y omiten los métodos y conocimientos educativos propios de contextos indígenas e interculturales (Harris, 2002; Quilaqueo \& Quintriqueo, 2015).

Ello se debe a que el problema se genera cuando el modelo tradicional de educación de corte occidental es legitimado como único; en tal contexto, las prácticas de enseñanza reposan sobre conocimientos occidentales, donde se utilizan metodologías de enseñanza poco participativas, descontextualizadas y con marcados énfasis magistocéntricos.

En concordancia con lo anterior, desde el contexto chileno Williamson y Flores (2015) señalan que el Programa de Educación Intercultural Bilingüe (Peib en adelante) no favorece la introducción de las lógicas de enseñanza indígenas dentro de la escuela y no contribuye a mejorar la enseñanza de las escuelas donde se implementa. En la misma dirección, Lagos (2015) evidencia que algunos educadores y educadoras interculturales carecen de una formación metodológica para enseñar lenguas indígenas. Desde esta perspectiva, cabe mencionar que estos profesores y profesoras difícilmente podrán vehiculizar los saberes y conocimientos de las culturas indígenas.

En este campo, desde la cultura mapuche los resultados de experiencias educativas en escuelas observadas en comunidades mapuche de la región de la Araucanía, Aylwin, Flanagan, Ermter y Muñoz (2005) expresan que son escasas las escuelas que plantean la pregunta sobre cómo enseñar en determinados contextos. En concordancia Leyton, Mancilla, Navarrete y Painen (2005, p. 69) evidencian que «si bien existe una valoración de algunos saberes de la cultura mapuche y se trata de incluir estos en el aula, existe evidentemente poco dominio de ello, como para llevarlo al contexto escolar». En tanto, es deseable que los profesores y profesoras, además de tener un dominio del conocimiento científico, dispongan de un conocimiento de los saberes culturales mapuche, y del mismo modo un conocimiento didáctico del contenido para movilizar la enseñanza de estos. En continuidad, en un estudio realizado por Quidel (2011), las estrategias metodológicas aplicadas para la enseñanza del Peib no responden al sistema de educación mapuche. Esto, porque la enseñanza de la lengua se realiza mediante las características que son propias y específicas de la cultura occidental. Se añade que el empleo de la metodología de enseñanza no se ajusta a la del mapuche, porque en ella va incluida la visión de mundo de las culturas occidentales. Asimismo, en una investigación realizada en el alto Bío-Bío, se observó que en las actividades y estrategias desarrolladas por la mayoría de los docentes no incluyeran elementos de la cultura mapuche (Acuña, 2013). Respecto a tales evidencias, cabe señalar que los profesores y profesoras que operan en contextos mapuche, reproducen el conocimiento occidental sobre la base de prácticas de enseñanza tradicionales centradas en el sujeto docente y por tanto descontextualizadas, pues excluyen elementos y saberes propios de la cultura mapuche, lo cual fragmenta la generación de aprendizajes significativos.

En consecuencia, desde los antecedentes expuestos es posible observar que la educación que se entrega en contextos indígenas e interculturales, y específicamente en el caso de contextos mapuche, se conservan prácticas educativas tradicionales enmarcadas en el conocimiento occidental y, por tanto, descontextualizadas. En tal sentido, se observa falta de dominio disciplinar respecto de métodos 
de enseñanza indígenas por parte de los profesores y profesoras, dada la débil o nula formación de docentes desde una educación intercultural. Esto se debe a la hegemonía cultural occidental de las formas de enseñanza. Lo anterior se sustenta en el predominio del positivismo y su enfoque conductista que ha llevado, por muchas décadas, a una enseñanza transmisionista con aprendizajes memorísticos que aminoran el conocimiento y uso de la didáctica en el aula (Nome, Nualart, Mansilla, \& Beltrán, 2013) desde una lógica contextualizada. Al respecto, Rodrigo y Arnay (1997) señalan que desde el positivismo lógico se hace hincapié en que sólo es posible una clase de conocimiento: el conocimiento científico occidental, el cual se ha instalado hace muchas décadas como un proyecto político y epistemológico legitimado por los Estados nacionales en la educación escolar.

Se espera que para superar en parte lo expuesto los sujetos docentes rompan con la forma tradicional de enseñanza, a fin de facilitar los procesos de transposición didáctica con base en estrategias y recursos didácticos y actividades contextualizadas. En este escenario, Quintriqueo, Torres, Gutiérrez y Sáez (2011) concluyen que el estudiantado aprende en la diferencia, considerando el conocimiento indígena y no indígena como una riqueza de la producción humana para mejorar la calidad de los aprendizajes, las oportunidades y la convivencia social. Al respecto, Quilaqueo y Torres (2013) sostienen que uno de los aspectos que pueden orientar la construcción de relaciones interculturales sustentadas en el conocimiento intercultural, es la incorporación de contenidos educativos y socioculturales indígenas en la escolarización para estudiantes de origen indígena y no indígena. Desde esta mirada, la educación intercultural en el contexto mapuche supone una contextualización de concepciones epistemológicas mapuche y no mapuche con base en los contenidos y finalidades educativas, para la formación de personas en el medio educativo (Quintriqueo \& Torres, 2013). La inclusión de procesos y metodologías de enseñanza de la cultura mapuche en el currículum escolar mejoraría la calidad de los aprendizajes y la convivencia social. En este campo, se hace necesario que el profesorado incorpore en sus prácticas de enseñanza no solamente el dominio de contenido disciplinario puro, sino además competencias asociadas a la apropiación del conocimiento didáctico para vehiculizar el saber disciplinario hacia el saber pedagógico, con el objeto de cumplir con el acto educativo (Pellón, Mansilla, \& San Martín, 2009). En este sentido, es esperable que el profesor o profesora tenga un dominio acabado de la transposición didáctica de contenido. Desde esta perspectiva, la transposición didáctica cobra relevancia, puesto que se requiere que el sujeto que ejerza la docencia porte un conocimiento amplio y profundo de cómo y cuándo debe enseñar, con una conducción apropiada de estrategias de enseñanza y aprendizaje, recursos didácticos e instrumentos de evaluación. Para ello, se debe tener en cuenta las características de los estudiantes y el contexto cultural.

A pesar de los esfuerzos por describir la transposición didáctica de saberes científicos en el contexto occidental, son escasas, sin embargo, las revisiones teóricas asociadas a la transposición didáctica de saberes culturales mapuche. Por tanto, en este artículo pretendemos llenar parte de ese vacío. A la luz de los antecedentes, el objetivo del trabajo a desarrollar es discutir sobre la transposición didáctica de saberes culturales mapuche que realizan los profesores y profesoras en escuelas situadas en contextos interculturales. Para ello, hacemos una revisión teórica del tema, en la cual desarrollamos una discusión basada en diferentes antecedentes teóricos que surgen de investigaciones y libros especializados.

En primer lugar, abordamos el método; en un segundo lugar conceptualizamos la didáctica y, junto con ello revisamos dos propuestas teóricas: el Conocimiento Didáctico del Contenido (CDC) y la transposición didáctica del contenido, al tiempo que abordamos la conceptualización y discusión teórica de las dos propuestas antes mencionadas. Luego realizamos una revisión de la transposición didáctica en contextos interculturales desde el campo internacional y chileno. Asimismo, hacemos una revisión de la transposición didáctica desde el contexto mapuche, en la que discutimos y describimos las relaciones entre saberes científicos y saberes culturales mapuche. Finalmente, este artículo remata con las consideraciones finales, de tal forma que nos permita visualizar y comprender la relevancia de la transposición didáctica y la manera en que se espera que esta opere en contextos mapuche e interculturales. 


\section{Metodología}

Para llevar a cabo la revisión teórica, consultamos diversas bases de datos y fuentes de indización internacional: WOS, Thomson Reuters, Scopus, Eric, Redalyc, Dianlet, Doaj y OEI, así como también libros especializados en campos relacionados con la temática.

Descartamos todos aquellos antecedentes teóricos y empíricos que no tuvieron relación directa con pueblos indígenas y el campo intercultural. Esto lo abordamos desde tres idiomas: español, francés, e inglés. Trabajamos con reportes de investigaciones y libros especializados; con estos procedimos a desarrollar la revisión del tema.

\section{Didáctica}

La didáctica es la disciplina que gobierna los procesos de enseñanza-aprendizaje; en tal sentido es necesario abordarla para entender el acto de transposición didáctica. En la década de los 70 la didáctica nace en rigor con el despertar de la enseñanza de los saberes en el campo de la escuela primaria del bachillerato y en las escuelas normales, que en lo sucesivo se ven confrontados con la formación disciplinar de las profesiones (Zambrano, 2006); a partir de tal circunstancia abordamos conceptualmente la didáctica.

Para Marhuenda (2000), la didáctica cobra todo sentido al ocuparse tanto de la selección y distribución del contenido, como de lo estrictamente curricular y la forma de enseñarlo. Es buscar unas técnicas, estrategias y recursos didácticos que permitan enseñar y lograr los objetivos de aprendizaje de una forma más efectiva. En correspondencia, Gallego y Salvador (2002, p. 108) señalan que «toda programación didáctica incluye una serie de elementos esenciales: (a) Los objetivos, (b) Los contenidos, (c) La metodología (estrategias didácticas), (d) Los recursos didácticos, y (e) La evaluación». Por lo tanto, la didáctica es una disciplina que incorpora una serie de dispositivos que permitan al sujeto docente enseñar saberes al estudiantado de forma eficaz. En este contexto, Medina (2002) señala que:

La didáctica ha evolucionado, en el sentido de que ha tendido a mejorar la comprensión de los procesos de enseñanza y aprendizaje. Apoyando a los estudiantes en su esfuerzo formativo y a los docentes en la mejora de su desarrollo profesional a partir del currículum, entendiéndose como un conjunto de elementos representativos de la contextualización y transformación práctica de las tareas formativas, entendidas en interacción emergente con la cultura (p. 22).

Al respecto, la didáctica desde el currículum contextualizado se ocupa de comprender de mejor forma la dinámica de los procesos de enseñanza y aprendizaje sobre la base de la integración de saberes científicos, culturales, metodológicos y actitudinales, para el proceso educativo y formativo del estudiantado. En este plano, para Beltrán, Mera, Moreno y Rodríguez (2007), la didáctica es la teoría de la enseñanza, y tiene por objeto el estudio del proceso de enseñanza de una forma integral, a saber: la instrucción, la enseñanza, las condiciones que propician el trabajo activo y creador del alumnado y su desarrollo intelectual. Respecto de tal concepción, la didáctica orienta la enseñanza a partir de la integración de conocimientos, mediante el uso de metodologías centradas en el alumno o alumna para vehiculizar el proceso de enseñanza y aprendizaje desde un plano activo, reflexivo, crítico y creativo. Además, la didáctica favorece la mejora del proceso de formación y educación en diversos contextos en que se lleve a cabo.

\section{Transposición didáctica del contenido y conocimiento didáctico del contenido}

La base de la didáctica es transversal y transdisciplinar, al ocuparse de los procesos de enseñanza y aprendizaje en toda situación formativo-instructiva que requiera de una comprensión integradora, 
holística e indagadora. Desde este campo disciplinar, surge la propuesta del Conocimiento Didáctico del Contenido (en adelante CDC), el cual se remonta a una conferencia que Lee Shulman pronunció en la Universidad de Texas, en Austin, durante el verano de 1983, titulada: «El paradigma perdido en la investigación sobre la enseñanza» (Shulman, 1987). Este paradigma resultó ser el pensamiento del profesor sobre el contenido del tema objeto de estudio y su interacción con la didáctica. Shulman (1999) postula que el centro de atención del contexto son las relaciones entre los agentes del «proceso didáctico» - profesor y alumno, y alumnos entre sí-. En esta interacción entre alumno y profesor convergen conocimientos que forman parte del proceso de enseñanza. En tal proceso subyacen conocimientos basales que forman parte del quehacer pedagógico del sujeto docente. Al respecto, Shulman (1987) señala que el conocimiento base para la enseñanza de un profesor o profesora, debe incluir al menos siete categorías de conocimiento diferentes: (i) conocimiento del contenido, (ii) conocimiento didáctico general, (iii) conocimiento curricular, (iv) conocimiento didáctico del contenido, (v) conocimiento de las características de los estudiantes y las estudiantes, (vi) conocimiento de los contextos educativos y (vii) conocimiento de las finalidades educativas, los valores educativos y los objetivos. En tanto, es preciso que el profesor o profesora integre en sus prácticas pedagógicas estos siete conocimientos, los que podrían facilitar su accionar en los procesos de enseñanza en contextos interculturales.

En este contexto, el CDC es un elemento central del conocimiento del profesor o profesora, y resulta fundamental hoy para promover el desarrollo profesional del profesorado en las disciplinas que desempeña. En conexión, Shulman (2005) describe al CDC como la comprensión de la materia por parte del sujeto docente que la transforma para hacerla «enseñable». En este sentido, el interés por el CDC implica un conjunto de saberes que permite al profesor o profesora trasladar a la enseñanza el contenido de un determinado tópico. Para Shulman (2005), el CDC representa la mezcla entre el contenido y la didáctica por la que se llega a la comprensión de cómo determinados temas y problemas se organizan, se representan y se adaptan a los diversos intereses del alumnado, y se exponen para la enseñanza.

Desde esta perspectiva, el conocimiento y comprensión que tenga el profesor o profesora sobre un conocimiento científico y sobre cómo adecuarlo a los intereses del estudiantado, facilita el proceso de enseñanza. Es decir, el CDC es una combinación adecuada entre el conocimiento de la materia a enseñar y el conocimiento pedagógico y didáctico relativo a cómo enseñarla (Marcelo, 2001). En tal sentido, es una combinación apropiada entre el conocimiento de la materia y el conocimiento didáctico sobre la base de metodologías de enseñanza pertinentes, las que posibilitarían organizar la enseñanza con más eficacia. Por lo tanto, la propuesta del Conocimiento Didáctico del Contenido (CDC) de Shulman (2005) cobra relevancia puesto que, además del conocimiento de la materia y del conocimiento general pedagógico, los profesores y profesoras deben desarrollar un conocimiento específico: cómo enseñar su materia específica, por lo que se requiere un CDC propio del buen hacer docente (Bolívar, 2005).

Respecto de tal fundamento, el sujeto docente debe incluir en sus prácticas pedagógicas modelos didácticos que faciliten la enseñanza de un determinado contenido. En ese sentido, el Instituto Tecnológico y de Estudios Superiores de Monterrey (Itesm) (2005), señala que, en la actualidad, resulta un reto para el profesorado el desarrollo de procesos didácticos que orienten el aprendizaje de los alumnos y alumnas, de tal manera que puedan responder a las demandas de la sociedad contemporánea. En concordancia con esa afirmación, el CDC moviliza medios didácticos para establecer enlaces entre el conocimiento disciplinar y el conocimiento didáctico del sujeto docente para facilitar la enseñanza en contextos diversos. En esta lógica, el CDC incluye las conexiones entre los conocimientos de la materia y los conocimientos didácticos del profesor o profesora (Acevedo, 2009). Esta interacción permite la transformación del contenido para su enseñanza (Chevallard, 1985), es decir, la transposición didáctica del contenido.

En ese campo, este autor plantea un concepto similar al del CDC: el de transposición didáctica. En relación con ese bosquejo, este estudio se fundamenta especialmente en la teoría llamada transposición 
didáctica «el saber sabio y el saber enseñado». Al respecto, Chevallard (1985) define la transposición didáctica como:

Un contenido del saber sabio que haya sido designado como [un] saber a enseñar sufre a partir de entonces un conjunto de transformaciones adaptativas que van a hacerlo apto para tomar lugar entre los objetos de enseñanza. El 'trabajo' que un objeto de saber a enseñar hace para transformarlo en un objeto de enseñanza se llama transposición didáctica (p. 39).

En conexión con la definición, el compromiso del profesor o profesora es transformar el saber científico en un saber enseñado utilizando para ello medios y modelos didácticos, con la finalidad de que el estudiantado pueda comprender ese lenguaje. La construcción de los saberes a enseñar y de los saberes enseñados/aprendidos, se muestra mucho más compleja que lo que dejan ver (Raisky \& Caillot, 1996). Tal dicho conlleva ciertas transformaciones en las formas de enseñar determinados contenidos.

En este contexto, se entiende al sujeto docente como aquella persona que construye un conocimiento acerca de la disciplina, y busca los sentidos, formas y medios para enseñar aquel contenido que pretende construir con sus alumnos y alumnas. El sujeto docente dispone de diversos conocimientos (Shulman, 2001) — presentes en todo momento en su quehacer profesional—, y media entre el conocimiento disciplinario y su enseñanza, mediación conocida también como transposición didáctica (Chevallard, 1985). En nuestra opinión, es esperable que el sujeto docente busque estrategias y recursos didácticos adecuados para llevar a cabo de buena forma los procesos de enseñanza y aprendizaje en determinados contextos educativos. En este sentido, Brousseau (1994) señala que el docente busca situaciones que den sentido a los conocimientos por enseñar. En esa lógica, el profesor o profesora debe contextualizar y recontextualizar los saberes, a fin de que el proceso de enseñanza tenga sentido para el estudiantado, de manera que se generen aprendizajes significativos.

\section{Transposición didáctica en contextos interculturales desde los campos internacional y chileno}

En relación con las evidencias empíricas y teóricas que se presentan en estudios realizados en el contexto indígena internacional, que han abordado la transposición didáctica del contenido, Vergara (2011) ha constatado que las prácticas educativas afrocolombianas están entrampadas en un sistema educativo que exige una alternativa metodológica, pedagógica y con distintas didácticas. En esta misma línea, en un estudio realizado en la región Ch’ol Chiapas por Bastiani, Ruiz-Montoya, Estrada, Cruz y Aparicio (2012), se concluye que durante la observación del trabajo en el aula, este se da en un ambiente de autoridad vertical, donde el sujeto docente decide las actividades que se realizarán sin mayor consideración de las capacidades y habilidades de sus estudiantes. También se observó que los profesores y profesoras no propician ambientes de trabajo nuevos y de mayor armonía. Las estrategias didácticas que empleó el equipo de docentes son convencionales: el profesor o profesora explica y asume el discurso como medio de comunicación y de interacción en el aula. Los alumnos y alumnas se quedan sentados en los pupitres escuchando a sus profesores. Respecto de tales experiencias, se visualizan estrategias didácticas centradas en el individuo docente, donde los estudiantes y las estudiantes tienen un rol pasivo, dado que no existen espacios para que estos reflexionen y generen aprendizajes.

Elías (2014) evidencia la falta de herramientas conceptuales y técnicas por parte de los sujetos docentes, las actitudes ambivalentes que expresan en torno a la utilización del guaraní y la persistencia de prácticas pedagógicas tradicionales que limitan el aprendizaje. Se evidencia así un débil dominio del Conocimiento del Contenido (CC) y del CDC. En contraste con lo anterior, el rol docente ha de estar focalizado en readecuar sus prácticas pedagógicas, centrándolas en la diversidad cultural y en las características del estudiantado, dado que según Lázar, Huber, Kriegler, lussier, Matei y Peck (2007), el profesor o profesora es determinante en la calidad de los procesos educativos de individuos estudiantes insertos en contextos de diversidad cultural. 
Por otro lado, las experiencias en la formación de estudiantes indígenas de Secundaria Comunitaria (SC) en Oxaca, México, según Ruiz y Quiroz (2014), han demostrado ventajas en su formación, puesto que estos grupos poblacionales construyen diferentes competencias. Además, se les forma cívica y moralmente para poder convivir en la diversidad cultural y lingüística, así como en el respeto hacia la naturaleza.

También es importante resaltar que este proyecto educativo ha logrado una mayor vinculación e interacción entre el estudiantado y la comunidad. El diálogo permanente que tienen los estudiantes y las estudiantes con las personas ancianas, con los padres y madres de familia, con los individuos informantes clave y con la autoridad local, son muestra de este logro. Asimismo, en un estudio realizado en Guatemala por Toledo y Guantá (2009), se concluye que las escuelas rurales que ofrecen programas de Educación Bilingüe Intercultural (EBI) superan a las escuelas rurales monolingües, pues obtienen una mejor comprensión de los aprendizajes por parte de las educandas y educandos. En este sentido, la tarea del sujeto docente es la de reformular y adecuar las prácticas pedagógicas, comprenderlas más allá del mero conjunto de estrategias para enseñar, y vincularlas a su verdadero significado. Ese cambio no puede lograrse simplemente con la formación del profesorado en las técnicas propuestas, en tanto es necesario convencer a los docentes y a las docentes de modificar los modelos de enseñanza a las distintas realidades educativas.

En Chile se han realizado algunos estudios sobre la transposición didáctica; uno de ellos fue elaborado por Romero (2004), en el cual se ha evidenciado que las estrategias didácticas empleadas por los profesores y profesoras son convencionales. Además, el alumnado señala que las clases son pasivas, que la pasan sentados y escribiendo; esta percepción también es compartida por los profesores y profesoras quienes expresan que se continúa trabajando de forma tradicional. Igualmente, un estudio realizado por Aylwin et al. (2005) revela que se pueden encontrar dificultades y escasez en el manejo de estrategias y de herramientas metodológicas y didácticas por parte del profesorado, es decir, docentes que no saben qué ni cómo enseñar. De tal evidencia se aprecia un escaso dominio del contenido disciplinar y de las estrategias didácticas necesarias para cumplir con el acto de enseñanza. Asimismo, se observa una escasez respecto de innovaciones metodológicas en los procesos didácticos. En esta realidad es preciso llevar a la práctica nuevas formas de dictar clases, de tal manera que se rompa con las clases verticales.

Otra investigación fue llevada cabo por Mansilla y Beltrán (2013); los resultados revelan que, en las prácticas pedagógicas de los profesores y profesoras se hallan incoherencias respecto de las estrategias didácticas que implementan en sus clases. Asimismo, se presentan incoherencias en la implementación de estrategias didácticas para generar resultados de aprendizajes. Tal hecho evidencia ciertas debilidades en el uso de la transposición didáctica del contenido.

Pasmanik y Cerón (2005) concluyen en su estudio que hay una exigua presencia de reflexión teórica en las clases, restándoles a los alumnos y alumnas la posibilidad de desarrollar las competencias propias del razonamiento. En correspondencia, en la investigación realizada por Nome et al. (2013) se constata que el modelo pedagógico dominante está centrado en el sujeto docente y se aproxima al modelo conductista. Respecto de tales experiencias, las prácticas del profesorado no constituyen una labor pedagógica sistemática ni enmarcada en paradigmas actuales de la educación, dado que sus prácticas pedagógicas se sustentan en modelos de enseñanza rígidos; es decir, emplean estrategias centradas en el sujeto docente y no en el individuo estudiante, las que se enmarcan en el paradigma conductista. En tanto, las prácticas pedagógicas que emplean los profesores y profesoras restringen el desarrollo del pensamiento crítico y reflexivo del estudiantado.

Cabe señalar que la reflexión cobra especial relevancia en los procesos de enseñanza y aprendizaje. Al respecto, Shulman (2005) plantea que la docencia se inicia cuando se reflexiona a fondo en torno a la enseñanza de algo, en torno a qué es lo que debe ser aprendido y cómo debe ser aprendido por los estudiantes y las estudiantes. En este sentido, la reflexión favorece el desarrollo de la práctica pedagógica del sujeto docente, facilita el desarrollo del pensamiento crítico de estudiantado, y por consiguiente mejora sus aprendizajes. Además, el sujeto docente debe tener una postura crítica y 
reflexiva respecto durante la preparación para la enseñanza, en su implementación, y una vez finalizada esta, a fin de mejorarla y adecuarla a los contextos en que se despliega.

Por consiguiente, la labor de la transposición didáctica involucra necesariamente reconocer, por una parte, las peculiaridades culturales de la disciplina desde donde se origina un objeto de saber, entendidas como la lógica disciplinar. Por otra, distinguir las peculiaridades de la cultura escolar desde donde se efectuará la transposición didáctica de dicho objeto de saber en objeto de enseñanza, entendida como lógica de la enseñanza y lógica del aprendizaje (Pellón et al., 2009). En tanto, la transposición didáctica del contenido debería incluir las características y estilos de aprendizaje de los alumnos y alumnas, como también reconocer sus saberes previos ubicados en contextos educativos culturales diversos, para generar nuevos aprendizajes, pues el sujeto adquiere conocimientos de su mundo en la medida en que es capaz de ir captando aquellas propiedades que lo caracterizan.

\section{Transposición didáctica desde el contexto mapuche}

La investigación llevada a cabo por Quintriqueo (2010) muestra que los profesores y profesoras en sus prácticas pedagógicas no establecen la relación entre las materias de enseñanza escolar y el saber propiamente mapuche. Asimismo, el estudio pone en evidencia que la educación escolar precisa de una relación educativa con el saber científico y con el conocimiento cultural mapuche para la formación de niños, niñas y adolescentes; es decir, se debe romper con la forma tradicional de enseñanza. En tal sentido, existe desconocimiento de cómo se dan los procesos de socialización primaria en esas comunidades, y de las lógicas que guían esos procesos de aprendizaje (Salgado, 2015).

En esta lógica, se requiere de estrategias de enseñanza y aprendizaje, y un currículum contextualizado para trasladar el conocimiento cultural mapuche a un objeto de enseñanza. Desde la perspectiva curricular, Beyer (2001) señala que el currículum constituye el eje de toda actividad educativa, por cuanto en su dimensión, tanto explícita como implícita, representa la esencia de la finalidad educativa y contenidos sustentados en marcos de referencias epistemológicos, éticos y culturales. Así, es preciso diseñar un currículum escolar intercultural, lo cual posibilitaría la presentación de contenidos educativos de una escuela que responda a grupos socioculturalmente diferenciados. Esto demanda que ciertas categorías de contenidos locales sean incorporadas en forma explícita en el currículum escolar (Essomba, 1999; Gimeno, 1995). En este sentido, se requiere la incorporación de saberes culturales mapuches al currículum escolar para transmitirlos a las nuevas generaciones con un sentido de pertinencia.

Desde este punto de vista, la transmisión de estos saberes permite a las nuevas generaciones desenvolverse en el marco de la sociedad que ha establecido un conjunto de disposiciones que la persona debe desarrollar en su identidad individual y social, en relación con los conocimientos de los patrimonios culturales mapuche y no mapuche (Bonfil-Batalla, 1988; Loslier, 1997; Quilaqueo \& Merino 2003; Quintriqueo \& Maheux 2004). Ello permitiría fortalecer la identidad sociocultural de los alumnos y alumnas de origen mapuche y no mapuche para aprender aspectos culturales mediante el estudio de saberes, costumbres, creencias y valores mapuche, favoreciendo el desarrollo de la interculturalidad entre ellos. En esta lógica, Cañulef (1998) señala que debe haber un re-pensamiento global de la educación, una nueva concepción del conocer, de las formas de pensar y de hacer escuela, de renovación y búsqueda de nuevas prácticas pedagógicas, de análisis y reflexión del quehacer educativo, con el fin de lograr la creatividad y la efectividad de los aprendizajes. Por lo tanto, es necesario que surjan espacios para el diálogo y la reflexión entre el estudiantado y el profesorado durante el desarrollo de las clases.

Surge entonces la «oralidad» como método de educación mapuche, la cual cobra especial relevancia, puesto que la educación mapuche tradicionalmente se desarrolla en el plano de la oralidad. Al respecto, según Llanquinao (2009) la oralidad: 
Es la comunicación verbal que se da en un contexto determinado, sistema por el cual los mapuches han generado formas eficaces de transmitir su visión de mundo, adecuadas a su realidad y que permiten proyectarse por medio de la palabra, que transforma y produce cambios en el oyente. (p. 222).

En esta línea, los mapuche sabios, es decir, los kimches ${ }^{1}$ priorizan estrategias que conlleven al aprender haciendo y aprender reflexionando (Quintriqueo et al., 2011). Los kimches desarrollan un trabajo cooperativo articulador desde perspectivas culturales diferentes que facilitan el desarrollo pedagógico en el aula.

Los kimches están dentro de la categoría de las personas ancianas —abuelas o abuelos-. Llanquinao (2009) señala que las personas mayores: los padres y madres, pero especialmente los abuelos y abuelas, son quienes van formando a los niños y niñas, guiándolos en el aprendizaje de los valores que se debe considerar a lo largo de su vida y que se debe mantener con la naturaleza, quienes mediante la palabra buscan reflexionar, para potenciar habilidades propias de cada niño y de cada niña, encontrando sus cualidades personales. En esta línea, Augusta (1903) señala que, en el caso de la cultura mapuche, los individuos poseedores del conocimiento son los ancianos. Asimismo, Pereira, Reyes y Pérez (2014), manifiestan que en el mundo mapuche las personas ancianas —abuelos y abuelas - son quienes han adquirido durante su vida la capacidad y la metodología para enseñar. Son capaces de entregar el kimün mapuche - conocimiento, saber mapuche - . En tal sentido, los abuelos y abuelas o los individuos ancianos, son los portadores del conocimiento y del saber mapuche, y son los encargados de transmitirlo a las nuevas generaciones; para ello, emplean una diversidad de metodologías y procesos de enseñanza y aprendizaje. Por lo tanto, es necesario implementar metodologías de enseñanza y aprendizaje que promuevan el trabajo colaborativo, el diálogo, el desarrollo del pensamiento reflexivo y crítico, la creatividad, el desarrollo de la autonomía, el aspecto valórico y ético del estudiante desde la cultura mapuche.

Desde esa perspectiva, es posible realizar la transposición didáctica según las exigencias que demanda la diversidad cultural y, por consiguiente, la sociedad contemporánea.

La transposición didáctica tiene pertinencia en la construcción de aprendizajes significativos, puesto que se produce dentro de variadas situaciones en las cuales los niños y niñas enfrentan verdaderos desafíos comunicativos y, por lo tanto, están conscientes del sentido y propósito de las actividades que realizan. Ello implicaría transformaciones en las prácticas pedagógicas de la docencia escolar, para lo cual es esencial provocar una verdadera crisis de manera de tensionar el paradigma dominante, a fin de dar paso al surgimiento de un nuevo paradigma con núcleos teóricos emergentes, ambicionando constituirse en ciencia normal (Kuhn, 1962). En tal sentido, se espera producir transformaciones de saberes sabios, lo cual implica cambios paradigmáticos en la forma de enseñar en contextos interculturales.

Desde el punto de vista epistemológico, los contenidos educativos ayudan a comprender y establecer rutas pedagógicas que permitan potenciar los procesos pedagógicos de los sujetos docentes, a fin de mejorar los procesos de transposición didáctica que tácitamente se producen en contextos interculturales.

En suma, en la actualidad resulta un reto para el profesorado el desarrollo de procesos didácticos que orienten el aprendizaje de los estudiantes y las estudiantes, de tal manera que puedan responder a las demandas de la sociedad actual. Esto implica transformaciones en las prácticas pedagógicas de los profesores y profesoras que operan en contextos interculturales, para mejorar la calidad de la educación de sus estudiantes, tanto en el aspecto educativo como formativo. Por ende, invitamos al profesorado a profundizar en una calidad de la enseñanza basada en la diversidad cultural.

Asimismo, los estados nacionales deben generar políticas educativas orientadas a la formación de docentes de calidad, a fin de que se desempeñen adecuadamente en contextos de diversidad cultural. Para ello se debe posicionar la interculturalidad desde un enfoque crítico, como un proyecto político y

1 El término hace referencia a la persona que porta los conocimientos sociales y culturales de la sociedad mapuche, que son vistos como tal dentro de sus comunidades. 
epistémico; se debe entender como una herramienta, como un proceso, un proyecto que se construye desde la gente como demanda para terminar con la subalternidad, la racialización y la discriminación que ejerce la cultura dominante que busca el desarrollo y creación de comprensiones y condiciones que no solo articulan y hacen dialogar las diferencias en un marco de legitimidad, dignidad, igualdad, equidad y respeto, sino que también y a la vez alientan la creación de modos «otros» ${ }^{2}$ de pensar, ser, estar, aprender, enseñar, soñar y vivir que cruzan fronteras (Walsh, 2009). Desde la lógica, es preciso abordar la descolonización de los saberes, pues De Sousa Santos (2010) sostiene que desde la diversidad epistemológica del mundo, debe existir el reconocimiento de una pluralidad de conocimientos más allá del saber científico. Esto implica renunciar a cualquier epistemología general, con el fin de terminar con la hegemonía cultural occidental como proyecto político presente en el currículum escolar.

Se hace necesario entonces diseñar un currículum intercultural, que permita dar respuesta a los conflictos detectados en contextos interculturales bajo el valor de la diversidad étnica, erradicando toda práctica discriminatoria, segregacionista o racista, y aplicando métodos didácticos creativos que propicien un cambio radical en las estructuras y en los significados de las relaciones humanas; por tanto, un cambio en toda la sociedad (González, 2011). En este sentido, Sartorello (2016a) señala que el currículum tendría que permitir al profesorado y al alumnado ampliar los conocimientos que se desprenden de las actividades que se realizan en la comunidad, articulándolas con los conocimientos escolares convencionales, para desarrollar una serie de habilidades y competencias que no se suele trabajar de forma sistemática en la pedagogía indígena. Esto implicaría pasar de lo propio a lo intercultural, por tanto los profesores y profesoras deben investigar y apropiarse de procesos de enseñanza y aprendizaje que se desarrollan en las comunidades indígenas, para luego incorporarlos al currículum escolar. Para ello se precisa una metodología coherente con la enseñanza de las lenguas originarias, así como estrategias didácticas que fomenten el inter-aprendizaje y la colaboración entre jóvenes (Sartorello, 2016b).

Lo expuesto anteriormente, en el caso del pueblo mapuche, permitiría reconocer, comprender, valorar y legitimar los saberes culturales mapuche desde un marco epistemológico sustentado en la cosmovisión de la cultura mapuche. Según (Mires, 1992, pp. 120-132), «la cosmovisión es el todo. Es la armonía, es el equilibrio, es el hombre, la naturaleza y lo que ocurre». Este marco está asociado a la comprensión que tienen los mapuche de la normativa cósmica, pues es la que determina el ordenamiento y comportamiento en la naturaleza, en la tierra, en los animales, en las personas, definiendo las formas o estilos de vida, las costumbres y normas adaptadas al wallmapu (territorio mapuche). La mayor preponderancia en esta interrelación la constituye el mundo espiritual, en su perfecta articulación entre la mente, el cuerpo, el espíritu y las emociones de la persona mapuche (Marileo, 2010).

Desde tal fundamento epistémico, es necesario avanzar hacia una transposición didáctica sustentada en saberes y conocimientos epistemológicos arraigados en la cosmovisión de la cultura mapuche, a partir del diálogo y el respeto, lo cual contribuiría a enriquecer y mejorar los procesos de formación y convivencia social de personas mapuches y no mapuches, dado que conviven e interactúan en una misma sociedad, pues el conocimiento del Otro (Pech, Rizo, \& Romeu, 2008) constituye una de las fuentes imprescindibles para la construcción de proyectos educativos y de vida en común, en contextos interculturales.

\section{Consideraciones finales}

En estas reflexiones finales aludimos a revisiones teóricas existentes en investigaciones y libros desde el campo internacional y chileno, donde el foco está puesto en la transposición didáctica de saberes culturales mapuche. Para ello, hemos abordado dos propuestas teóricas: la propuesta del

2 Hablar de modos «otros» es tomar distancia de las formas de pensar, saber, ser y vivir inscritas en la razón occidental-colonial. 
Conocimiento Didáctico del Contenido (CDC) y la transposición didáctica del contenido. En cuanto a la propuesta CDC de Shulman (2005), este modelo propone, por un lado, la comprensión de la materia por parte del sujeto docente, y por otro, la transformación para hacerla «enseñable». En este sentido, el modelo resulta interesante debido a que el CDC implica un conjunto de saberes que permite al profesor o profesora trasladar a la enseñanza el contenido de un determinado tópico.

En esta misma línea, Chevallard (1985) desarrolla una propuesta teórica similar al del CDC, y es la teoría de la transposición didáctica, «el saber sabio y el saber enseñado». Estas propuestas teóricas se enfocan en el contexto intercultural, desde el cual proporcionan ideas sobre cómo un conocimiento científico y los saberes culturales mapuche, a partir de la utilización de una serie de medios didácticos, se convierten en elementos aptos para la enseñanza.

A partir de la discusión y reflexión de los estudios revisados, constatamos que la transposición didáctica de saberes culturales mapuche se dificulta debido a los procesos de enseñanza rígidos, enmarcados en el paradigma conductista y descontextualizados, lo que restringe el empleo del diálogo, de la reflexión y de la elaboración de significados por parte de estudiantado. En concordancia, se tiende a omitir los aportes de los estudiantes y las estudiantes, y por consiguiente la construcción de aprendizajes. En tal sentido, estamos de acuerdo con De Miguel-Díaz (2005), quien expresa que para realizar la transposición didáctica del conocimiento no sólo es importante el conocimiento disciplinario, sino también las particularidades de la cultura escolar. Tal idea implica no alejarse del conocimiento del estudiantado inserto en contextos culturales mapuche, con lo que se lograría una adecuada transposición didáctica del saber cultural mapuche.

Al respecto, es preciso — según Quintriqueo (2010) — que los profesores y profesoras repliquen estrategias metodológicas con un enfoque intercultural. La articulación entre el conocimiento cultural mapuche y el conocimiento escolar científico requiere un trabajo colaborativo, liderazgo y diálogo entre los sujetos docentes y los miembros de las comunidades mapuche - especialmente los ancianos y ancianas-, como herramientas de cambio en la enseñanza. En concordancia, se requiere adecuaciones curriculares y didácticas que aporten a la enseñanza de contenidos pertinentes a las características sociales y culturales que moldean al estudiantado. En tal sentido, es posible aplicar un modelo didáctico integrador y transformador con base en la transposición didáctica de los saberes culturales mapuche sustentada en los fundamentos epistemológicos, desde una postura de diálogo, respeto y equidad. Este argumento permitiría avanzar en los procesos de la transposición didáctica que se desarrollan en contextos interculturales; para ello se requiere de docentes que dispongan de un conocimiento amplio y profundo sobre cómo y cuándo se debe enseñar, con una conducción apropiada de estrategias de enseñanza y aprendizaje y con recursos didácticos e instrumentos de evaluación, para lo cual se debe tener en cuenta el contexto. Desde esta postura, se espera una mejora continua del proceso de enseñanza y aprendizaje, y la convivencia social entre niños y jóvenes mapuches y no mapuches, sobre la base de un currículum flexible y contextualizado en relación con las características y necesidades educativas de estos, y así superar la monoculturalidad occidental que impera en la educación para alumnos y alumnas de origen tanto mapuche como no mapuche (Quilaqueo, Quintriqueo, \& San Martín, 2011); y asimismo, terminar con la hegemonía cultural occidental a la que están sujetos los diversos pueblos indígenas. Por lo tanto, es urgente profesionalizar la docencia en diversos contextos culturales, con el objetivo de preparar ciudadanas y ciudadanos críticos y reflexivos, con la connotación de que los sujetos apliquen tales conocimientos en la vida cotidiana, y ello les permita insertarse y desenvolverse adecuadamente en la sociedad actual desde una perspectiva integral.

En consecuencia, nos parece fundamental estudiar los procesos de transposición didáctica que realizan los profesores y profesoras en contextos interculturales, con la finalidad de buscar medios didácticos para vehiculizar de mejor forma el proceso de enseñanza y aprendizaje. Esto permitiría, por un lado, comprender el desarrollo de los procesos didácticos y, por otro, avanzar en el conocimiento de la transposición didáctica que se lleva a cabo en contextos interculturales. 


\section{Lista de referencias}

Acevedo, J. (2009). Conocimiento didáctico del contenido para la enseñanza de la naturaleza de la ciencia (I): el marco teórico. Revista Eureka sobre Enseñanza y Divulgación de las Ciencias, 6(1), 21-46.

Acuña, L. (2013). Contextualización curricular y visibilización pewenche: significados atribuidos por docentes de primer ciclo básico a la implementación del currículum oficial en sus prácticas pedagógicas de aula en alto Bio-Bío. (Tesis para optar al grado de Magíster en Educación, mención en Currículum y Comunidad Educativa). Universidad de Chile, Santiago de Chile, Chile.

Akkarai, A. (2009). Introducción aux aproches interculturales en éducation. Génova: Universidad de Génova.

Augusta, F. (1903). Lecturas araucanas. Temuco: Editorial Kushe.

Aylwin, M., Muñoz, L., Flanagan, A., \& Ermter, K. (2005). Buenas prácticas para una pedagogía efectiva. Santiago de Chile: Unicef.

Bastiani, J., Ruiz-Montoya, L., Estrada, E., Cruz, T., \& Aparicio, J. (2012). Práctica docente, castellanización, burocracia y centralización de la educación como limitaciones del éxito pedagógico en la región Ch'ol, Chiapas. Perfiles Educativos, 34(135), 8-25. Recuperado de: http://www.scielo.org.mx/pdf/peredu/v34n135/v34n135a2.pdf

Beltrán, L., Mera, E., Moreno, J., \& Rodríguez, G. (2007). Estrategias didácticas desarrolladas por los docentes para orientar el trabajo integrador en la facultad de Psicología. Revista de Psychología, (1), 133-162.

Beyer, L. (2001). El currículo en conflicto. Madrid: Akal.

Bolívar, A. (2005). Conocimiento didáctico del contenido y didácticas específicas. Profesorado. Revista de Currículum y Formación del Profesorado, 9(2), 1-39. Recuperado de: https://www. ugr.es/ recfpro/rev92ART6.pdf

Bonfil-Batalla, G. (1988). La teoría del control cultural en el estudio de los procesos. Brasilia: Universidad de Brasilia, Tempo Brasileiro.

Brousseau, G. (1994). Los diferentes roles del maestro. Barcelona: Paidós.

Cañulef, E. (1998). Hacia la interculturalidad y el bilingüismo en la educación chilena. Temuco: Conadi.

Chevallard, Y. (1985). Le transposition didactique. Du savoir savan tau savoir enseigné. Grenoble: La Pensée Sauvage.

Cochran-Smith, M., \& Fires, K. (2005). The Area on research and teacher education: Contexts on goals. En M. Cochran-Esmith, \& K. Zeichner (Eds.), Estudyng teacher education. The report of the Area panel of research on teacher education, (pp. 37-68). New Jersey: Laurence Erlbaum Associates.

Darling-Hamond, L. (2000). Teacher quality and student achievement. Review of State Police. Educational Police Analysis Archives, 8(1), 1-49. Recuperado de: https://epaa.asu.edu/ojs/ article/view/392/515

De Miguel-Díaz, M. (2005). Cambio de paradigma metodológico en la educación superior. Exigencias que conlleva. Cuadernos de Integración Europea, 2, 16-27.

De Sousa-Santos, B. (2010). Descolonizar el saber, reinventar el poder. Montevideo: Trilce. Recuperado de: http://www.boaventuradesousasantos. pt/media/Descolonizar\%20el\%20saber final $\% 20-\% 20 \mathrm{C} \% \mathrm{C} 3 \%$ B3pia.pdf

Elías, R. (2014). Fundamentos de la educación bilingüe y sus implicancias en la práctica docente. Revista electrónica Umbral, (7), 1-13. Recuperado de: http://www.reduc.cl/wp-content/ uploads/2014/08/edubili.pdf

Essomba, M. (1999). Construir la escuela intercultural. Reflexiones y propuestas para trabajar la diversidad étnica cultural. Barcelona: Grao. 
Gallego, J., \& Salvador, F. (2002). El diseño didáctico: objetivos y fines. En M. Antonio, \& F. Salvador (Eds.), Didáctica general, (pp.105-127). Madrid: Pearson Educacion.

Gimeno, J. (1995). El currículum: una reflexión sobre la práctica. Madrid: Morata.

González, E. (2011). La interculturalidad: implicaciones educativas. En F. J. García-Castaño, \& N. Kressova (Coords.), Actas del I Congreso Internacional sobre Migraciones en Andalucía, (pp. 697-702). Granada: Instituto de Migraciones.

Gordon-Burns, D., \& Campbell, L. (2014). Indigenous rights in Aotearoa/New Zealand Inakitia rawatia hei kakano mō āpōpō: Students' encounters with bicultural commitment. Childhood Education, 90, 20-28. doi:https://doi.org/10.1080/00094056.2014.872506

Harris, H. (2002). Coyote goes to school: The paradox of indigenous higher education. Canadian journal of native education, 26(2), 96-187. Recuperado de: https://eric.ed.gov/?id=EJ665139

Horst, C., \& Gitz-Johansen, T. (2010). Education of ethnic minority children in Denmark: Monocultural hegemony and counter positions. Intercultural Education, 21(2), 137-151. doi:https://oi. org/10.1080/14675981003696271

Instituto Tecnológico y de Estudios Superiores de Monterrey (Itesm). (2005). Ejemplo de técnicas y estrategias didácticas. Recuperado de: http://www.uctemuco.cl/docencia/pioneros/docs/apoyo/ Ejemplos\%20de\%20estrategias\%20 y\%20tecnicas\%20didacticas.pdf

Kuhn, T. (1962). La Estructura de las revoluciones científicas. México, D. F.: Fondo de Cultura Económica.

Lagos, C. (2015). El Programa de Educación Intercultural Bilingüe y sus resultados: ¿perpetuando la discriminación? Pensamiento Educativo. Revista de Investigación Educacional Latinoamericana, 52(1), 84-94. Recuperado de: http://pensamientoeducativo.uc.cl/files/journals/2/articles/676/ public/676-2138-1-PB.pdf

Lázar, I., Huber-Kriegler, M., Lussier, D., Matei, G., \& Peck, Ch. (2007). Développer et évaluer la competence en comunicación interculturelle; un guide à l'usage des enseignants de langues et des formateurs d'enseignants. Estrasburgo: Éditions du Conseil de l' Europe.

Leyton, M., Mancilla, P., Navarrete, V., \& Painen, P. (2005). Noción de Educación Intercultural Bilingüe en experiencias educativas de dos escuelas ubicadas en comunidades mapuche de la IX región. (Tesis de licenciatura en educación). Universidad Católica de Temuco, Temuco, Chile.

Loslier, S. (1997). Des relations interculturelles. Quebec: Editions Liber.

Llanquinao, H. (2009). Valores de la educación tradicional mapuche: posibles contribuciones al sistema educativo chileno. (Tesis doctoral inédita). Universidad de Barcelona, Barcelona, España.

Mansilla, S., \& Beltrán, J. (2013). Coherencia entre las estrategias didácticas y las creencias curriculares de los docentes de segundo ciclo, a partir de las actividades didácticas. Perfiles Educativos, 35(139), 25-39.

Marhuenda, F. (2000). Didáctica general. Madrid: De la Torre.

Marcelo, C. (2001). El aprendizaje de los formadores en tiempos de cambio. La aportación de las redes y el caso de la Red Andaluza de Profesionales de la Formación. Profesorado. Revista de Currículum y Formación de Profesorado, 5(1), 1-17.

Marileo, A. (2010). ¿Quiénes somos los mapuche? Latitudes latinas. Recuperado de: http:// latitudeslatinas.com/download/artigos/Qui\%C3\%A9nes\%20somos\%20los\%20Mapuche.pdf

Medina, A. (2002). La didáctica: disciplina pedagógica aplicada. En M. Antonio, \& F. Salvador (Eds.), Didáctica general, (pp. 5-31). Madrid: Pearson Educación.

Mires, F. (1992). El Discurso de la Indignidad. Quito: Abya-Yala.

Nome, C., Nualart, Z., Mansilla, J., \& Beltrán, J. (2013). Representaciones que poseen los profesionales del área de la salud respecto de sus prácticas de enseñanza de las asignaturas disciplinarias en el aula. Revista Facultad de Medicina-Universidad Nacional de Colombia, 61(1), 17-23.

Organización para la Cooperación y el Desarrollo Económico (Ocde). (2005). Teachers matter: attracting, developing and retaining effective teachers. Paris: Ocde. 
Pasmanik, D., \& Cerón, R. (2005). Las prácticas pedagógicas en el aula como punto de partida para el análisis del proceso enseñanza-aprendizaje: un estudio de caso en la asignatura de química. Estudios Pedagógicos, 31(2), 71-87. doi:http://dx.doi.org/10.4067/S0718-07052005000200005

Pech, C., Rizo, M., \& Romeu, V. (2008). Manual de comunicación intercultural. México, D. F.: Universidad Autónoma de la Ciudad de México.

Pellón, M., Mansilla, J., \& San Martín, D. (2009). Desafíos para la transposición didáctica y conocimiento didáctico del contenido en docentes de anatomía: obstáculos y proyecciones. International Journal of Morphology, 27(3), 743-750. doi:http://dx.doi.org/10.4067/S071795022009000300018

Pereira, J., Reyes, M., \& Pérez, F. (2014). Ecos de las pablaras de la tierra desde un último confin del mundo. Reencontrando el ser mapuche pewenche en el valle del Queuco. Santiago de Chile: Talleres de Andros.

Pineau, P. (2001). ¿Por qué triunfó la escuela? o la modernidad dijo: «esto es educación», y la escuela respondió: «yo me ocupo». En P. Pineau (Ed.), La escuela como máquina de educar. Tres escritos sobre un proyecto de la modernidad, (pp. 27-52). Buenos Aires: Paidós.

Quilaqueo, D., \& Merino, M. (2003). Estereotipos y prejuicio étnico hacia los mapuches en textos complementarios a la asignatura de Historia. Revista Campo Abierto, (23), 119-135. Recuperado de: https://dialnet.unirioja.es/ejemplar/89078

Quilaqueo, D., \& Quintriqueo, S. (2015). Métodos educativos mapuche: retos de la doble racionalidad educativa: aportes para un enfoque de educación intercultural. Temuco: Ediciones Universidad Católica de Temuco.

Quilaqueo, D., Quintriqueo, S., \& San Martín, D. (2011). Contenido de aprendizajes educativos mapuches para el marco conceptual de un currículum escolar intercultural. Estudios Pedagógicos, 37(2), 233-248. doi:http://dx.doi.org/10.4067/S0718-07052011000200014

Quilaqueo, D. \& Torres, H. (2013). Multiculturalidad e interculturalidad: desafíos epistemológicos de la escolarización desarrollada en contextos indígenas. Alpha, (37), 285-300. doi:http://dx.doi. org/10.4067/S0718-22012013000200020

Quintriqueo, S. (2010). Implicancias de un modelo curricular monocultural en contexto mapuche. Santiago de Chile: Gráficas Lom.

Quintriqueo, S., \& Maheux, G. (2004). Exploración del conocimiento sobre la relación de parentesco como contenido educativo para un currículum escolar intercultural en comunidades mapuche. Revista de Psicología, 13(1), 73-91. Recuperado de: file://C:/Users/beltr/ Downloads/17488-1-51473-1-10-20111209\%20(1).pdf

Quintriqueo, S., Morales, S., Quilaqueo, D., \& Arias, K. (2016). Interculturalidad para la formación inicial docente: desafios para construir un diálogo intercultural. Temuco: Ediciones Universidad Católica de Temuco.

Quintriqueo, S., \& Torres, H. (2013). Construcción de conocimiento mapuche y su relación con el conocimiento escolar. Estudios Pedagógicos, 39(1), 199-216. doi:http://dx.doi.org/10.4067/ S0718-07052013000100012

Quintriqueo, S., Torres, H., Gutiérrez, M., \& Sáez, D. (2011). Articulación entre el conocimiento cultural mapuche y el conocimiento escolar en ciencia. Educción. Educadores, 14(3), 475-492.

Raisky, C., \& Caillot, M.(1996). Doit-on en finir avec la transpositiondidactique? essai de contribution à une théoriedidactique. Paris, Bruxelles: De BoeckUniversité.

Rodrigo, M. J. \& Arnay, J. (1997). La construcción del conocimiento escolar. Barcelona: Paidós.

Romero, A. (2004). La jornada escolar completa ¿una política para la calidad y equidad de la educación? La JEC desde la mirada de los protagonistas de una Comunidad Educativa. (Tesis de magíster inédita). Universidad de Chile, Santiago de Chile, Chile.

Ruiz, A., \& Quiroz, E. (2014). Educación comunitaria: una propuesta alternativa para los pueblos indígenas de Oaxaca-México. Revista Polis, 13(38), 225-24. doi:http://dx.doi.org/10.4067/ S0718-65682014000200011 
Salgado, N. (2015). La diversidad en la construcción de mundo de niños y niñas de dos culturas. Revista Latinoamericana de Ciencias Sociales Niñez y Juventud, 13(1), 357-368. doi:http:// dx.doi.org/10.11600/1692715x.13121260914

Sartorello, E. (2016a). La co-teorización intercultural de un modelo educativo en Chiapas, México. Quito: Abya-Yala.

Sartorello, E. (2016b). Convivencia y conflicto intercultural: jóvenes universitarios indígenas y mestizos en la universidad intercultural de Chiapas. Revista Mexicana de Investigación Educativa, 21(70), 719-757. Recuperado de:

http://www.scielo.org.mx/pdf/rmie/v21n70/1405-6666-rmie-21-70-00719.pdf

Shulman, L. (1987). Knowledge and teaching: foundations of the new reform. Harvard Educational Review, 57(1), 1-22.

Shulman, L. (1999). Ways of thinking, ways of teaching. New York: Teachers College Press.

Shulman, L. (2001). Conocimiento y enseñanza. Estudios Públicos, (83) 163-196.

Shulman, L. (2005). Conocimiento y enseñanza: fundamento de la nueva reforma. Profesorado. Revista de Currículum y Formación de Profesorado, 9(2), 1-30. Recuperado de: https://www. ugr.es/ recfpro/rev92ART1.pdf

Toledo, M., \& Guantá, M. (2009). Modelo bilingüe intercultural. Ciudad de Guatemala: Ministerio de Educación de Guatemala.

Vergara, I. (2011). Prácticas educativas que evidencian la enseñanza de la afrocolombianidad en contextos interculturales en el trabajo del aula de los docentes etnoeducadores en las instituciones educativas de la ciudad de Pereira. (Tesis inédita de maestría). Universidad Tecnológica de Pereira, Pereira, Colombia.

Walsh, C. (2009). Interculturalidad crítica y educación intercultural. Recuperado de: file://C:/Users/ beltr/Downloads/interculturalidad\%20critica\%20y\%20educacion\%20intercultural\%20(10).pdf

Williamson, G., \& Flores, F. (2015). Estado del arte de la Educación Intercultural Bilingüe en Chile, 1990-2013. Temuco: Universidad de la Frontera.

Zambrano, L. (2006). Tres tipos de saber del profesor y competencias: una relación compleja. Revista Educere, 10(33), 225-232. Recuperado de: http://www.redalyc.org/articulo.oa?id=35603303 